\title{
AVALIAÇÃO DA LORDOSE DA COLUNA CERVICAL NOS PACIENTES COM ESCOLIOSE DO TIPO LENKE I SUBMETIDOS AO TRATAMENTO CIRÚRGICO
}

\author{
EVALUATION OF CERVICAL BEHAVIOR IN PATIENTS WITH SCOLIOSIS LENKE WHO \\ UNDERWENT SURGERY
}

\author{
EVALUACIÓN DE LA LORDOSIS DE LA COLUMNA CERVICAL EN LOS PACIENTES CON \\ ESCOLIOSIS DEL TIPO LENKE I SOMETIDOS AL TRATAMIENTO QUIRÚRGICO
}

José Lucas Batista Junior ${ }^{1}$, Priscila Rossi de Batista², Diogo Miranda Barbosa ${ }^{3}$, Igor Cardoso Machado ${ }^{4}$, Rodrigo Rezende ${ }^{5}$

\begin{abstract}
RESUMO
Objetivo: Avaliar a angulação da lordose cervical nos pacientes portadores de Escoliose Idiopática do Adolescente (EIA) do tipo Lenke I, no período pré- e pós-operatório. Método: Estudo prospectivo e descritivo em que foram avaliados pacientes com ElA, sendo documentadas medidas antropométricas. Foram avaliadas as angulações das radiografias (posição ortostática) em perfil cervical de C3 a C7 no período pré- e pós-operatório, estabelecendo como normal a lordose cervical entre 10 e 30 graus. Resultados: Participaram 26 pacientes com EIA, sendo a maioria do sexo feminino (73\%), com média de idade de 14,3 anos. Observamos que no período pré-operatório 80,9\% dos pacientes apresentavam angulação cervical não fisiológica, sendo que 42,3\% dos pacientes apresentavam diminuição da lordose cervical $\left(<10^{\circ}\right)$ e $38,6 \%$, inversão desta angulação $\left(<0^{\circ}\right)$. No pós-operatório, todos os pacientes apresentaram melhora da angulação cervical em relação aos níveis fisiológicos, sendo que 69,3\% dos pacientes obtiveram a angulação normal desejada da coluna cervical e nenhum dos pacientes manteve inversão da lordose cervical. Conclusões: A correção cirúrgica da escoliose cursou com melhoria secundária da angulação da lordose cervical.
\end{abstract}

Descritores: Coluna vertebral; Doenças da coluna vertebral; Escoliose; Lordose; Radiografia.

\begin{abstract}
Objective: This study was to evaluate the angle of cervical lordosis in patients with Adolescent ldiopathic Scoliosis (AlS) ranked in Lenke I pre- and postoperatively. Methods: Prospective and descriptive study, which evaluated patients with AlS and documented anthropometric measures. We assessed the angulations of the radiographs (orthostatic position) in cervical profile of C3 to C7 in the pre- and post-operative setting as the normal cervical lordosis between 10 and 30 degrees. Results: A total of 26 patients with AlS, the majority being female (73\%) with mean age of 14.3 years. We noted that in the pre-operative $42.3 \%$ of patients had reduced cervical lordosis $\left(<10^{\circ}\right)$ and $38.6 \%$, reversal of this angle $\left(<0^{\circ}\right)$. Postoperatively, all patients had improvement in cervical angle in relation to physiological levels, with $69,3 \%$ of patients achieving the desired normal angle and no cervical spine of patients maintained reversal of cervical lordosis. Conclusions: Surgical correction of scoliosis has led to the secondary improvement of angulation of the cervical lordosis,
\end{abstract}

Keywords: Spine; Spinal diseases; Scoliosis; Lordosis; Radiography.

\section{RESUMEN}

Objetivo: Evaluar el ángulo de la lordosis cervical en pacientes con Escoliosis Idiopática del Adolescente (EIA), clasificados en Lenke I en el pre y posoperatorio. Métodos: Estudio prospectivo y descriptivo, en el que se evaluó a pacientes con ElA y se documentaron mediciones antropométricas. Se evaluó la angulación de las radiografías (posición ortostásica) de perfil de columna cervical de C3 a C7, en el entorno de pre y posoperatorias, estableciéndose como normal la lordosis cervical entre 10 y 30 grados. Resultados: Un total de 26 pacientes fue evaluado, en su mayoría mujeres (73\%) con edad promedio de 14,3 años. Observamos que, en el período preoperatorio, 80,9\% de los pacientes presentaban angulación cervical no fisiológica, siendo que 42,3\% de los pacientes habían reducido la lordosis cervical (<10 ) y 38,6\% la inversión de este ángulo $\left(<0^{\circ}\right)$. Después de la operación, todos los pacientes tuvieron una mejoría en el ángulo de la columna cervical en relación con los niveles fisiológicos, con 69,3\% de los pacientes que alcanzaron el ángulo normal deseado de la columna cervical y ninguno de los pacientes mostró una reversión de la lordosis cervical. Conclusiones: La corrección quirúrgica de la escoliosis produjo una mejora secundaria de la angulación de la lordosis cervical.

Descriptores: Columna vertebral; Enfermedades de la columna vertebral; Escoliosis; Lordosis; Radiografía.

1. Ortopedista eTraumatologista Especialista em Cirurgia da Coluna Vertebral, Médico Assistente do Grupo de Coluna Vertebral da Santa Casa de Vitória - Vitória, ES, Brasil.

2. Fisioterapeuta Assistente do Grupo de Coluna Vertebral da Santa Casa de Vitória - Vitória, ES, Brasil.

3. Radiologista Especialista em Sistema Músculo-Esquelético, Chefe da Residência em Radiologia do Centro de Diagnóstico por Imagem (CDI) - Vitória, ES, Brasil.

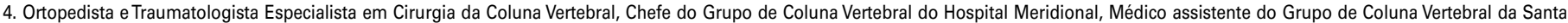
Casa de Vitória - Vitória, ES, Brasil.

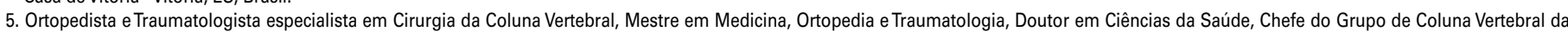
Santa Casa de Vitória, Chefe do Grupo de Coluna Vertebral do Vila Velha Hospital - Vitória, ES, Brasil.

Trabalho realizado no Hospital Meridional - Cariacica, Espírito Santo, Vila Velha, Hospital Vila Velha, Espírito Santo e Hospital Santa Casa de Misericórdia de Vitória, Vitória, E S, Brasil. Correspondência: Rodrigo Rezende. Rua Desembargador Augusto Botelho, 209/801 - Praia da Costa, Vila Velha - ES, Brasil. CEP: 29101-110. E-mail: grupodecoluna @ santacasavitoria.org 


\section{INTRODUÇÃO}

A escoliose é definida como uma deformidade tridimensional, na qual no plano frontal apresenta desvio lateral maior de 10 graus. Esta deformidade pode cursar com assimetria dos ombros e do triângulo do talhe, presença de giba costal proeminente na região da convexidade da curva e alterações do equilíbrio sagital ${ }^{1-9}$.

Alcançar um adequado equilíbrio sagital é cada vez mais importante para o tratamento cirúrgico da EIA, pois a correção desta deformidade deve ser realizada nos três planos, evitando desta forma que se crie deformidades secundaria como flat back e possibilitando um melhor equilíbrio sagital. Embora a ênfase no passado fosse a correção do plano coronal, tornou-se evidente que a saúde em longo prazo da coluna vertebral pode ser mais relacionada com um adequado equilíbrio no plano sagital ${ }^{10-12}$

Em relação ao plano sagital, grande parte dos pacientes com EIA apresentam hipocifose torácica e inversão da lordose cervical, alterações estas que podem influenciar no tratamento e na qualidade de vida pós operatória destes pacientes. A inversão da lordose cervical quando não corrigida pode ocasionar alterações degenerativas precoce, aumentando a incidência de cervicalgia ${ }^{1-5,10-12}$

Por considerarmos que o equilíbrio sagital cervical adequado, é imprescindível na evolução satisfatória de pacientes portadores de escoliose idiopática do adolescente é que realizamos este estudo com objetivo de avaliar a angulação da lordose cervical no período pré e pós-operatório.

\section{MÉTODOS}

Foram avaliados 26 pacientes portadores de EIA, sendo documentada a idade, peso e altura. Foi utilizado o método de Cobb para mensuração da lordose cervical, A medida das angulações foram realizadas em radiografias panorâmicas da coluna vertebral em ortostase incluindo os níveis C3 a C7 no período pré e pósoperatório (Figura 1).

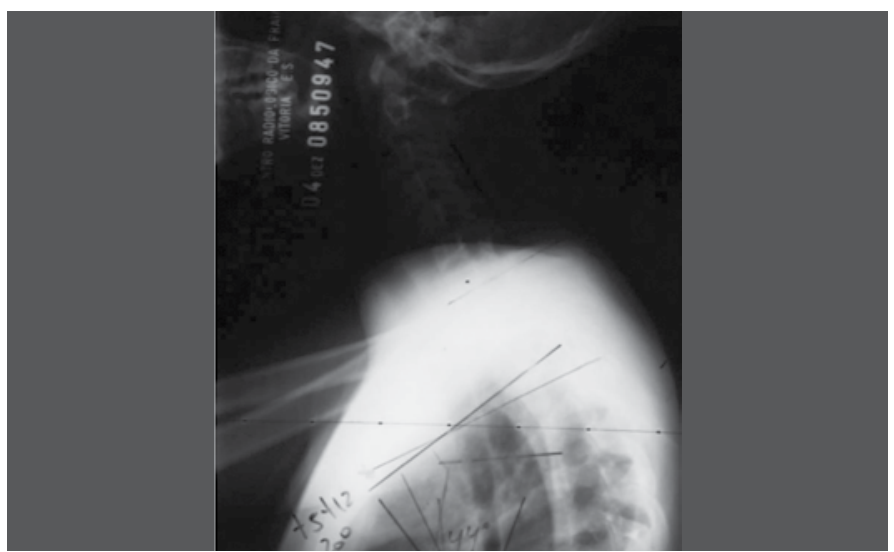

Figura 1. Método de Cobb para aferição da angulação cervical (C3-C7).

O valor fisiológico da lordose cervical (C3 a C7) foi considerado normal entre 10 e 30 graus, hipolordose entre 0 a 10 graus e como inversão da lordose as angulações abaixo de 0 grau $^{16,17}$

No critério de inclusão foram selecionados pacientes portadores de EIA, de ambos os sexos, em período pré-operatório de correção cirúrgica da deformidade vertebral, classificados, segundo Lenke, em tipo I, que fazem acompanhamento no grupo de coluna da Santa Casa de Misericórdia de Vitória e do Vila Velha Hospital.

Foram excluídos, pacientes portadores de EIA que já tiveram sido submetidos à cirurgia para correção de EIA, bem como os pacientes sem indicação cirúrgica e outros tipos de escoliose. Também foram excluídos do estudo pacientes que, em algum momento apresentaram algum processo patológico associado à EIA, e que não pertenciam à classificação de Lenke do tipo I
Os resultados de idade, peso e altura foram representados por números através de valores de média \pm desvio padrão (DP), sendo o gênero expressado em porcentagem. A comparação entre as médias obtidas de angulação da lordose cervical no pré e pós-operatório foi analisada estatisticamente através de teste $t$ de Student pareado, com significância dada para valores de $p<0,05$.

A análise dos dados foi realizada utilizando os softwares Microsoft Office / Excel 2007 e GraphPad Prism (San Diego, CA, EUA).

\section{RESULTADOS}

Participaram desta pesquisa 26 pacientes portadores de EIA, dos quais 19 eram do sexo feminino (73\%) e 7 do sexo masculino (27\%), com uma idade média de 14,3 anos. O peso médio encontrado na amostra foi de $46,2 \mathrm{Kg}$, a altura média de 1,59m (Tabela 1).

A média da angulação da lordose cervical pré-operatória foi de 4,2 graus e a pós-operatória de 17,8 graus. Este aumento significativo da angulação nos permite evidenciar uma aproximação da lordose cervical para níveis mais próximos do normal diante da correção cirúrgica da escoliose. Encontramos 80,9\% dos pacientes com diminuição da lordose cervical $\left(<10^{\circ}\right)$ ou inversão desta angulação $\left(<0^{\circ}\right)$ (Figura 2).

Após intervenção cirúrgica, todos os pacientes estudados apresentaram melhora da angulação cervical, sendo que nenhum manteve inversão da lordose cervical. No pós-operatório, 69,3\% dos pacientes obtiveram a angulação fisiológica desejada da coluna cervical, preconizada entre $10^{\circ}$ a $30^{\circ}$ (Tabela 2).

Muito embora tenha ocorrido aumento da angulação lordótica cervical para valores mais próximos do normal, do total de pacientes operados, $19,2 \%$ ainda apresentavam hipolordose $\left(0^{\circ}\right.$ a $\left.10^{\circ}\right) \mathrm{e}$ $11,5 \%$ obtiveram lordose acima do esperado $\left(>30^{\circ}\right)$.

Tabela 1. Característica da amostra.

\begin{tabular}{c|c}
\hline Parâmetros & Média \pm DP \\
\hline Idade (anos) & $14,3 \pm 1,9$ \\
\hline Peso $(\mathrm{Kg})$ & $46,2 \pm 12,3$ \\
\hline Altura $(\mathrm{m})$ & $1,59 \pm 0,1$ \\
\hline IMC $\left(\mathrm{Kg} / \mathrm{m}^{2}\right)$ & $18,02 \pm 2,9$ \\
\hline
\end{tabular}

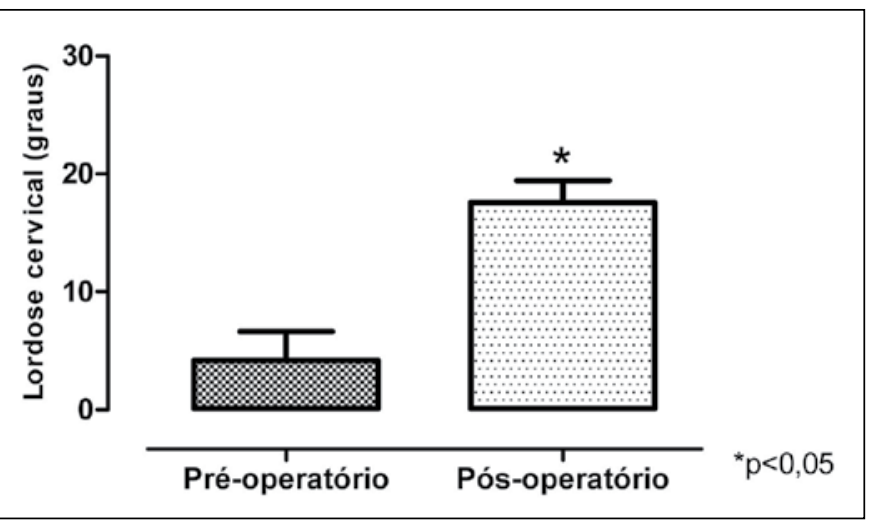

Figura 2. Comparação da angulação da lordose cervical no período pré e pós-operatório de correção cirúrgica da deformidade de pacientes com Escoliose Idiopática do Adolescente (EIA).

Tabela 2. Avaliação da angulação cervical pré e pós-operatório de pacientes submetidos a tratamento cirúrgico de escoliose.

\begin{tabular}{|c|c|c|c|c|}
\hline & $\begin{array}{c}\text { Inversão } \\
\left(\mathbf{A}^{\circ}<\mathbf{0}\right)\end{array}$ & $\begin{array}{c}\text { Hipolordose } \\
\left(\mathbf{0}>\mathbf{A}^{\circ}<\mathbf{1 0}\right)\end{array}$ & $\begin{array}{c}\text { Fisiológica } \\
\left(\mathbf{1 0}>\mathbf{A}^{\circ}<\mathbf{3 0}\right)\end{array}$ & $\begin{array}{c}\text { Hiperlordose } \\
\left(\mathbf{3 0}>\mathbf{A}^{\circ}\right)\end{array}$ \\
\hline $\begin{array}{c}\text { Pré-operatório } \\
(\mathrm{N}=26)\end{array}$ & $\begin{array}{c}38,6 \% \\
(\mathrm{~N}=10)\end{array}$ & $\begin{array}{c}42,3 \% \\
(\mathrm{~N}=11)\end{array}$ & $\begin{array}{c}19,1 \% \\
(\mathrm{~N}=5)\end{array}$ & $0 \%$ \\
\hline $\begin{array}{c}\text { Pós-operatório } \\
(\mathrm{N}=26)\end{array}$ & $0 \%$ & $\begin{array}{c}19,2 \% \\
(\mathrm{~N}=5)\end{array}$ & $\begin{array}{c}69,3 \% \\
(\mathrm{~N}=18)\end{array}$ & $\begin{array}{c}11,5 \% \\
(\mathrm{~N}=3)\end{array}$ \\
\hline
\end{tabular}




\section{DISCUSSÃO}

Até a década de 50 , os princípios do tratamento cirúrgico das deformidades vertebrais baseavam-se na realização de artrodese posterior in situ seguida de repouso no leito e imobilização gessada por seis meses. Harrington, nos anos 60, foi o primeiro a utilizar materiais especiais para a correção das escolioses onde a instrumentação utilizava haste metálica e ganchos ${ }^{18,19}$. Em 1977, Luque associou ao sistema de Harrington os fios sublaminares, permitindo um maior potencial de correção e não necessitando da utilização de gesso no pós-operatório ${ }^{20}$. Apesar dessa grande evolução dos sistemas de fixação para o tratamento cirúrgico das deformidades, havia apenas a correção em um único plano, sendo observada, no pós-operatório destes pacientes, a presença de retificação da coluna torácica conhecida como flat back, associando-se a uma evolução não satisfatória, causada pelo desequilíbrio sagital.

Devido a estas alterações no plano sagital, diversos estudos foram realizados no intuito de se evitar a manutenção ou a piora do equilíbrio sagital em pacientes submetidos a cirurgia de correção de escoliose ${ }^{10,21}$. Dentre estes estudos, Cotrel-Dubousset et $\mathrm{al}^{21}$ criaram, na década de 80 , um novo sistema de fixação da coluna vertebral chamado de terceira geração de implantes, em que se tornou possível corrigir não apenas a deformidade no plano frontal como também a deformidade no plano sagital ${ }^{19}$.

Foi a partir de Cotrel e Dubousset et al. ${ }^{21}$ que a importância do equilíbrio sagital normal tornou-se reconhecida no tratamento de pacientes com deformidades vertebrais, estudos comprovam que um adequado balanço sagital da coluna vertebral influencia de forma positiva a qualidade de vida dos pacientes acometidos com EIA, em especial na biomecânica das articulações sacroilíacas, quadris, joelhos e tornozelos 22,23

Já o desequilíbrio no plano sagital pode levar à dor crônica e incapacidade ${ }^{24}$, as alterações da lordose cervical, da cifose torácica e da lordose lombar normal tem sido associada a quadros álgicos e de degeneração discal acelerada ${ }^{25,26,}$ corroborando para a importância do equilíbrio sagital adequado buscado em nosso trabalho.

Encontramos em nosso estudo que a grande maioria dos pacientes $(80,3 \%)$ encontravam-se com medidas cervicais não fisiológicas no período pré-operatório e que após o tratamento cirúrgico houve grande melhora desta angulação, dos quais $69,3 \%$ dos pa- cientes obtiveram angulação fisiológica da coluna cervical. Nenhum dos pacientes permaneceu com inversão da lordose cervical fato este que demonstra que é possível a correção secundária da angulação cervical através da correção da cifose torácica por este fato cirurgiões devem estar atentos não apenas para a correção no plano frontal mas também no sagital.

No resultado do tratamento cirúrgico da EIA, considera-se fundamental a análise do equilíbrio sagital, em que a angulação cervical deve ser avaliada e corrigida no momento do tratamento cirúrgico ${ }^{11}$. Devido à presença de hipocifose torácica nos pacientes com escoliose ocorre uma retificação ou inversão da angulação fisiológica cervical ${ }^{11,12,24,25}$. Já foi descrito na literatura que a correção da cifose torácica leva a um melhor equilíbrio do tronco com melhora da angulação cervical, evitando a evolução do processo degenerativo discal ${ }^{11,25,26}$.

Cochran et al. ${ }^{27}$ e Moskowitz et al. ${ }^{28}$ avaliaram seus pacientes, no período pós-operatório, com retificação da coluna torácica em um longo follow up. Observaram que muitos desses pacientes evoluíam com dor cervical secundária a um desequilíbrio sagital, devido à inversão da lordose cervical. Em nosso trabalho a maioria dos pacientes obtive a lordose cervical fisiológica restabelecida após a cirurgia de correção de EIA

Acreditamos que a realização do nosso trabalho possa contribuir para despertar o interesse de novos estudos sobre a importância do plano sagital nestas deformidades, visto que existem poucos trabalhos na literatura em que se estuda o comportamento da coluna cervical no plano sagital destes pacientes.

Julgamos que o restabelecimento da cifose torácica fisiológica no tratamento cirúrgico das deformidades vertebrais é de extrema importância, pois através dele é possível a correção secundária da angulação cervical, contribuindo para um melhor equilíbrio sagital, consequentemente melhora da qualidade de vida destes pacientes.

\section{CONCLUSÃO}

O tratamento cirúrgico de pacientes portadores de escoliose idiopática do adolescente tipo Lenke I, através da correção da curva torácica produziu uma melhora secundária da angulação cervical, estabelecendo um melhor equilíbrio sagital.

\section{REFERÊNCIAS}

1. Carr WA, Moe JH, Winter RB, Lonstein JE. Treatment of idiopathic scoliosis in the Milwaukee brace. J Bone Joint Surg Am.1980;62(4):599-612.

2. Takemitsu Y, Harada Y, Ando M. Anteroposterior spinal curvature of normal growing children. J Jpn Orthop. Assoc. 1977;51:795- 801

3. Voutsinas SA, MacEwen GD. Sagittal profiles of the spine. Clin Orthop Relat Res. 1986;(210):235-42

4. Bunnell WP. The natural history of idiopathic scoliosis. Clin Orthop Relat Res. 1988;(229):20-5

5. Cowell HR, Hall JN, MacEwen GD. Genetic aspects of idiopathic scoliosis. A Nicholas Andry Award essay, 1970. Clin Orthop Relat Res. 1972;86:121-31.

6. Bridwell KH, Betz R, Capelli AM, Huss G, Harvey C. Sagittal plane analysis in idiopathic scoliosis patients treated with Cotrel-Dubousset instrumentation. Spine (Phila Pa 1976). 1990;15(7):644-9.

7. Crawford $\mathrm{AH}$, Macke J, Claudle R. Sagital plane correction by contoured Harrington rods and interspinous segmental instrumentation. Orthos Trans 1989;13:238-44.

8. De Smet AA, Asher MA, Cook LT, Goin JE, Scheuch HG, Orrick JM. Threedimensional analysis of right thoracic idiopathic scoliosis. Spine (Phila Pa 1976). 1984;9(4):377-81.

9. Ecker ML, Betz RR, Trent PS, Mahboubi S, Mesgarzadeh M, Bonakdapour A, et al. Computer tomography evaluation of Cotrel-Dubousset instrumentation in idiopathic scoliosis. Spine (Phila Pa 1976). 1988;13(10):1141-4.

10. Booth KC, Bridwell KH, Lenke LG, Baldus CR, Blanke KM. Complications and predictive factors for the successful treatment of flatback deformity (fixed sagittal imbalance). Spine (Phila Pa 1976). 1999;24(16):1712-20.

11. Hilibrand AS, Tannenbaum DA, Graziano GP, Loder RT, Hensinger RN. The sagittal alignment of the cervical spine in adolescent idiopathic scoliosis. J Pediatr Orthop. 1995:15(5):627-32.

12. Knight, RQ, Jackson, RP, Killian JT, Stanley EA. Scoliosis Research Society: White Paper on Sagittal Plane Alignment. Avaliable at www.srs.org/profissonals.

13. Batzdorff $U$, Batzdorff A. Analysis of cervical spine Curvature in patients with cervical spondylosis. Neurosurgery. 1988;22(5):827-36.

14. Hellsing E, Reigo T, McWilliam J, Spangfort E. Cervical and lumbar lordosis and thoracic kyphosis in 8, 11 and 15-year-old children. Eur J Orthod. 1987;9(2):129-38.

15. Rechtman Am, Boreadis Borden AG, Gershon-Cohen J. The lordotic curve of the cervical spine. Clin Orthop. 1961;20:208-16.

16. Jackson RP, Peterson MD, McManus AC, Hales C. Compensatory spinopelvic balance over the hip axis and better reliability in measuring lordosis to the pelvic radius on standing lateral radiographs of adult volunteers and patients. Spine (Phila Pa 1976). 1998;23(16):1750-67.

17. Korovessis PG, Stamatakis MV, Baikousis AG. Reciprocal angulation of vertebral bodies in the sagittal plane in an asymptomatic Greek population. Spine (Phila Pa 1976). 1998:23(6):700-4.

18. Green NE. The role of Harrington rods and Wisconsin wires in Idiopathic scoliosis. In: Bridwell KH, DeWald RL, editors. The textbook of spinal surgery. 2nd ed. St. Louis: Lippincott; 1996: p.469.

19. Harrington PR. Treatment of scoliosis: correction and internal fixation by spine instrumentation. June 1962. J Bone Joint Surg Am. 2002;84(2):316.

20. Luque ER. Segmental spinal instrumentation for correction of scoliosis. Clin Orthop Relat Res. 1982;(163):192-8.

21. Cotrel $Y$, Dubousset J. [A new technic for segmental spinal osteosynthesis using the posterior approach]. Rev Chir Orthop Reparatrice Appar Mot.1984;70(6):489-94.

22. Stagnara P, De Mauroy JC, Dran G, Gonon GP, Costanzo G, Dimnet J et al. Reciprocal angulation of vertebral bodies in a sagittal plane: approach to references for the evaluation of kyphosis and lordosis. Spine (Phila Pa 1976).1982;7(4):335-42.

23. Wambolt A, Spencer DL. A segmental analysis of the distribution of lumbar lordosis in the normal spine. Orthop Trans. 1987;11:92-3.

24. Jang JS, Lee SH, Min JH, Kim SK, Han KM, Maeng DH. Surgical treatment of failed back surgery syndrome due to sagittal imbalance. Spine (Phila Pa 1976). 2007:32(26):3081-7.

25. Boseker EH, Moe JH, Winter RB, Koop SE. Determination of "normal" thoracic kyphosis: a roentgenographic study of 121 "normal" children. J Pediatr Orthop. 2000;20(6):796-8.

26. Propst-Proctor SL, Bleck EE Radiographic determination of lordosis and kyphosis in normal and scoliotic children. J Pediatr Orthop. 1983:3(3):344-6.

27. Cochran T, Irstam L, Nachemson A. Long term anatomic and functional changes in patients with adolescent idiopathic scoliosis treated by Harrington rod fusion. Spine (Phila Pa 1976) 1983;8(6):576-83.

28. Moscowitz A, Moe JH, Winter RB, Binner H. Long term follow-up of scoliosis fusion. J Bone Joint Surg Am. 1980;62:364-75. 